\title{
Synthesis of hydroxylated group IV metal oxides inside hollow graphitised carbon nanofibers: nano-sponges and nanoreactors for enhanced decontamination of organophosphates.
}

\begin{abstract}
Maxwell A. Astle, ${ }^{a}$ Graham A. Rance, ${ }^{\mathrm{a}, \mathrm{b}}$ Michael W. Fay, ${ }^{\mathrm{b}}$ Stuart Notman, ${ }^{\mathrm{c}}$ Mark R. Sambrook, ${ }^{\mathrm{c}}$ and Andrei N. Khlobystov*,a,b.

The confinement and enhanced catalytic properties of hydroxylated group IV metal oxide nanostructures inside hollow graphitised carbon nanofibers (GNF) has been demonstrated. GNF - a structural analogue of carbon nanotubes - were effectively filled with suitable precursor molecules of metal chlorides from the gas and liquid phases. Subsequent basecatalysed hydrolysis afforded amorphous, nanostructured hydroxylated metal oxide $\left(\mathrm{MO}_{\mathrm{x}}(\mathrm{OH})_{\mathrm{y}}\right.$ where $\mathrm{M}=\mathrm{Zr}, \mathrm{Ti}$, and $\left.\mathrm{Hf}\right)$ thin films, which coat the internal surfaces of GNF. This versatile and general strategy allows the chemical composition and morphology of the encapsulated material to be modified by varying the conditions used for hydrolysis and post-synthesis thermal treatment. The increased Lewis acidic properties and high surface area of the zirconium composite promote the catalysed hydrolysis of dimethyl nitrophenyl phosphate (DMNP) - a toxic organophosphorus chemical. A four-fold enhancement in the rate of DMNP hydrolysis relative to its separate constituent components was observed, highlighting the surprising synergistic abilities of this composite material to perform both as a 'nano-sponge', absorbing the harmful compounds inside the GNF, and a nanoreactor, enhancing the local concentration of organophosphate around the hydroxylated metal oxide species, leading to improved catalytic performance.
\end{abstract}

\section{Introduction}

Carbon nanotubes have recently emerged as excellent containers for atoms, molecules and the broadest range of lowdimensional materials, attracting particular attention owing to their ability to act as nanoreactors, where the unique effects of spatial confinement at the nanoscale enable control of reaction pathways..$^{1-4}$ The remarkable capability of carbon nanotubes to contain chemical reactions originates from their superior chemical, mechanical and thermal stability relative to more traditional nanoscale containers, such as molecular capsules and porous solids. 5,6 Moreover, the encapsulation of materials inside carbon nanotubes provides a universal platform, distinct from other nanocontainers, as carbon nanotubes rely predominantly on ubiquitous van der Waals interactions to drive encapsulation. ${ }^{7}$

Amongst carbon nanotubes, hollow graphitised carbon nanofibers (GNF) are particularly suitable for application as catalytic nanoreactors, due to their large internal diameter, high chemical robustness and unique internal morphology (Figure 1). ${ }^{8}, 9$ Whilst the hydrophobic internal channels of carbon nanotubes allow for reactants to be readily drawn into the lumen from solution, GNF, being significantly wider than typical carbon nanotubes, additionally permit the efficient diffusion of products from the internal channel, making them particularly effective as nanoreactors. Furthermore, the 3-4 nm high stepedges on the interior surfaces of GNF are understood to provide exemplary anchoring points for guest-materials, with previous studies demonstrating that small metallic nanoparticle catalysts can be effectively formed at these sites. ${ }^{10,}{ }^{11}$ Moreover, the corrugated interior of the GNF facilitates the maximisation of host-guest interactions, often leading to significant enhancements in activity and selectivity, as well as improved stability and recyclability of the confined nanocatalyst. This has been shown across a range of preparative catalytic reactions, including the Suzuki-Miyaura cross-coupling and hydrogenation reactions. ${ }^{11,12}$ Interestingly, though not explored to date, the nanofiber interior has the additional potential to act as template, thus promoting the formation of unique topologically complex nanostructures with larger surface areas that can be used to further drive improved catalytic performance.

Several techniques have been previously employed for filling hollow carbon nanostructures with metal species, including the gas-phase decomposition of metal carbonyl or metal acetylacetonate precursors, for the direct synthesis of transition metal nanoparticles within carbon nanotubes. ${ }^{13,} 1415$ Nanostructured metal oxides can subsequently be formed by oxidising the metal nanoparticles at high temperature in air; however, this often leads to the development of larger and more crystalline particles with a consequently lower surface area and reduced catalytic activity. ${ }^{16}$ Some metal halides, as potential precursors to metal oxides, have previously been inserted into nanotubes using a capillary filling method and used to probe the nature of host-guest interactions. ${ }^{17}$ However, the confinement of group IV metal oxides inside carbon

a. School of Chemistry, University of Nottingham, University Park, NG7 2RD, UK

b. Nanoscale and Microscale Research Centre, University of Nottingham, University

Park, NG7 2RD, UK.

CBR Division, Defence Science and Technology Laboratory (Dstl), Porton Down, Salisbury, Wiltshire, SP4 OJQ, UK.

Electronic Supplementary Information (SI) available: [Supplementary characterization analysis for $\mathrm{Ti}, \mathrm{Zr}$, $\mathrm{Hf}$ oxide composite materials, including surface area, metal: oxygen ratio and further microscopy data. Additional hydrolysis data with an assessment of catalyst post-decontamination]. See DOI: $10.1039 / x 0 x x 00000 x$ 

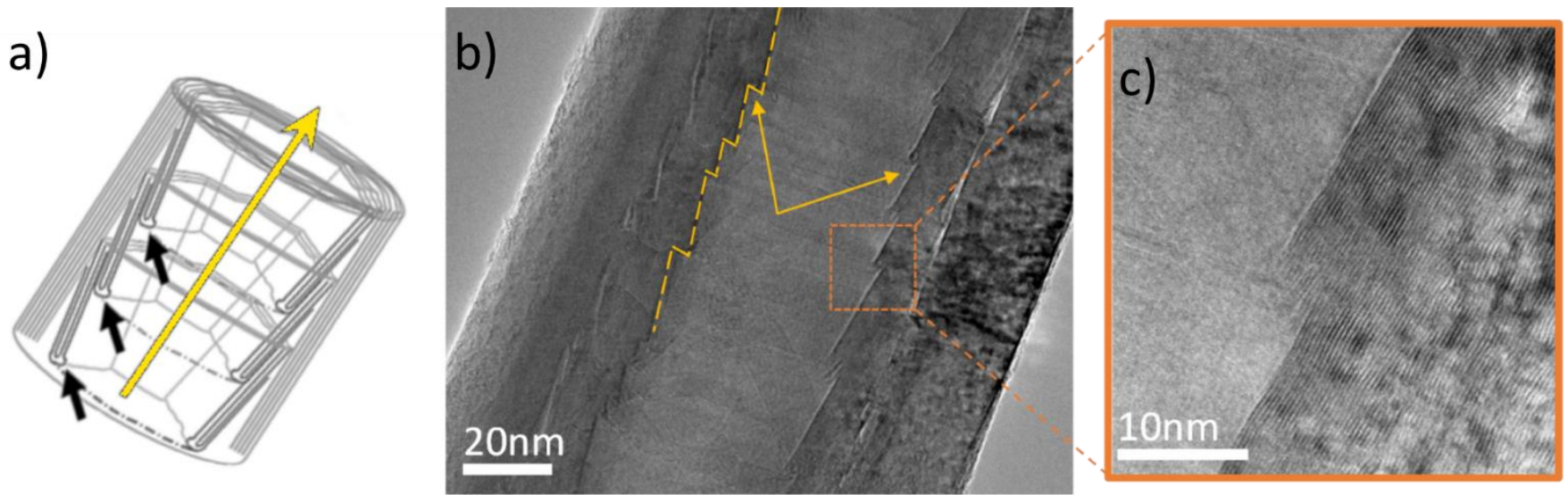

Figure 1. (a) Schematic representation of the structure of GNF (interior step-edges are denoted by black arrows; the yellow arrow signifies the direction of the nanofiber growth axis). (b,c) Transmission electron microscopy images highlighting the large internal diameter and unique structure of GNF. The interior stepedges, emphasised in (c), act as anchoring points for atoms, molecules, and nanomaterials due to increased van der Waals interactions between the confined guest and the host-nanofiber at these locations. ${ }^{11}$

nanotubes remains largely unexplored, thus negating investigation of the catalytic properties of these promising materials in nanoreactors. The challenge for the formation of metal oxides inside nanotubes is particularly topical because early transition metal oxides (especially group IV metal oxides and hydroxides) have low toxicity, high availability and chemical robustness. ${ }^{18-21}$ Moreover, they possess low coordination numbers, high Lewis acidity of metal sites, and different types of hydroxide groups on their surface, and thus exhibit remarkable catalytic performance in the oxidation, reduction and hydrolysis of organic molecules. 22,23-27 For example, zirconium hydroxide has shown impressive rates of decontamination for organophosphorus (OP) agents, with the active surface groups able to affect both the adsorption and hydrolysis of these toxic compounds. ${ }^{28-30}$ OP species, containing phosphate and/or thiolophosphate ester bonds, represent some of the most toxic chemicals in the world, posing a substantial and potentially imminent threat to human health. ${ }^{31-}$ 34

Enhancement of the catalytic performance of group IV metal oxides by combining their unique catalytic properties with the nanoscale confinement effects offered within nanoreactors, particularly in the context of catalytic decontamination of OP compounds, presents a topical and important research challenge. In this study, we developed a highly effective method for the synthesis of nanostructured group IV metal oxides by hydrolysis of corresponding metal chlorides directly within the cavity of GNF. Thus formed hybrid materials of amorphous hydroxylated $\mathrm{Ti}, \mathrm{Zr}$ or $\mathrm{Hf}$ oxides, which coat the inner surface of nanoreactors, lead to the enhanced catalytic performance of the metal hydroxide in the hydrolysis of OP compounds.

\section{Experimental}

\section{General}

Standard reagents and solvents, including the simulant dimethyl nitrophenyl phosphate ( $97 \%$ purity), were purchased from Sigma-Aldrich Chemicals (Dorset, UK) and were used asreceived. Graphitised nanofibers (PR19-XT-HHT, Fe content
$<100 \mathrm{ppm})$ were obtained from Pyrograf Products Inc (Cedarville, Ohio).

Thermogravimetric analysis (TGA) was performed using a TA Q500 thermogravimetric analyser. All samples were deposited onto platinum pans for analysis, heated in air ( $90 \mathrm{~mL} / \mathrm{min})$ from room temperature up to $1000^{\circ} \mathrm{C}$ at a rate of $5^{\circ} \mathrm{C} / \mathrm{min}$, followed by an isothermal hold at $1000{ }^{\circ} \mathrm{C}$ for $10 \mathrm{~min}$. All powder X-ray diffraction (PXRD) measurements were performed on a PANalytical X'Pert Pro diffractometer equipped with a $\mathrm{Cu} K(\alpha)$ radiation source $(\lambda=1.5432 \AA, 40 \mathrm{kV}, 40 \mathrm{~mA})$ in Bragg-Brentano geometry. For ambient temperature measurements, the sample was mounted on a Si zero-background holder using isopropyl alcohol to aid adhesion. The parameters for a typical experiment were: $5^{\circ}$ start angle, $80^{\circ}$ stop angle, $0.0525^{\circ}$ step size, $6080 \mathrm{~s}$ time per step and $0.00220 \%$ s scan speed. Hightemperature PXRD measurements were performed using an Anton Parr (HTK 1200N) high-temperature oven chamber in the air up to $600{ }^{\circ} \mathrm{C}$ on an alumina support. Significant background alumina support peaks were removed to exaggerate sample peaks. Surface area analysis was performed using the BrunauerEmmett-Teller (BET) method based on adsorption data in the relative pressure $(\mathrm{P} / \mathrm{Po})$ range from 0.02 to 0.22 by measuring nitrogen sorption isotherms of the samples at $-196^{\circ} \mathrm{C}$ on a Micromeritics ASAP 2020 sorptomter. Samples were degassed for 16 hours at $100^{\circ} \mathrm{C}$.

Transmission electron microscopy (TEM) and dark field scanning transmission electron microscopy (STEM) were performed using a JEOL JEM-2100+ microscope operated at 200 $\mathrm{kV}$. TEM samples were prepared via a drop casting technique, where samples were dispersed in methanol, followed by deposition onto copper grid mounted "lacey" carbon films (Agar). All images were processed using Gatan Digital Micrograph software. Energy dispersive X-ray (EDX) spectroscopy and mapping were acquired using an Oxford Instruments INCA X-ray microanalysis system. Ka values were used for carbon, oxygen chlorine and titanium, the $L \alpha$ value was used for zirconium and the M $\alpha$ value was used for hafnium. The beam was condensed to areas suspended over holes of the amorphous carbon film to negate the contribution to the 
carbon signal from the support film. Electron energy loss spectroscopy (EELS) was performed using an Enfinium SE system. Tomography measurements were conducted using a JOEL FEGTEM microscope operating at $200 \mathrm{keV}$ and using a Gatan 916 high tilt tomography holder, acquiring images at $1^{\circ}$ tilt intervals over a range of -40 to +40 degrees with gold nanoparticles as fiducial markers. Annular dark-field images were acquired at $10 \mathrm{~cm}$ camera length. Tomographic reconstruction was performed via the IMOD v4.9.3 software, using 10 iterations of the SIRT algorithm and an approach proposed by DN Mastronarde (1997). ${ }^{35}$ Scanning electron microscopy (SEM) was performed on a JEOL 6490LV SEM operating at $15 \mathrm{kV}$.

UV-Vis absorption spectra were recorded at room temperature using $1 \mathrm{~cm}$ quartz cuvettes. The samples were analysed using a Perkin-Elmer Lambda $25 \mathrm{UV} /$ Vis spectrometer at a scan rate of $240 \mathrm{~nm} / \mathrm{min}$ over a wavelength range of 200-500 $\mathrm{nm}$. Spectra were analysed using UV WinLab ES software.

Preparation of hydroxylated metal oxides inside graphitised nanofibers

For the liquid-phase filling of $\mathrm{TiCl}_{4}$ into graphitised nanofibers, GNF (25 mg) were pre-treated at $500{ }^{\circ} \mathrm{C}$ for $45 \mathrm{~min}$ in air to remove any materials, such as adsorbed water or residual amorphous carbon, which could otherwise block the internal channel. The annealed GNF were then immediately placed into a Schlenk tube under an inert atmosphere of argon and heated to $80^{\circ} \mathrm{C}$. $\mathrm{TiCl}_{4}(1 \mathrm{~mL})$ was added and the mixture stirred at 500 rpm for 1 hour. The obtained slurry was then exposed to air and, once the production of hydrochloric acid vapour had subsided, washed with ammonium hydroxide $(5 \mathrm{~mL}, 35 \%)$ with gentle agitation.

For the gas-phase filling $\mathrm{ZrCl}_{4}$ and $\mathrm{HfCl}_{4}$ into graphitised opened, with the resultant black solid washed with ammonium hydroxide ( $5 \mathrm{~mL}, 35 \%)$ with gentle agitation.

For all materials, ethyl acetate $(5 \mathrm{~mL})$ was then added and the biphasic mixture vigorously shaken. The aqueous layer was removed and discarded, with the remaining black solid collected from the organic layer by vacuum filtration $(0.2 \mu \mathrm{m}$ PTFE membrane) and dried under ambient conditions overnight.

\section{Hydrolysis reactions of DMNP}

Hydrolysis experiments were performed at room temperature. In a typical experiment, the composite material $(23.25 \mathrm{mg}$, containing $1.25 \mathrm{mg}$ of $\mathrm{ZrO}_{\mathrm{x}}(\mathrm{OH})_{\mathrm{y}}$ ) was suspended in potassium hydroxide solution ( $2 \mathrm{~mL}, 1 \mathrm{mM}, \mathrm{pH} 10$ ) with gentle agitation. DMNP $(8.0 \mu \mathrm{L}, 0.05 \mathrm{mmol})$ was added to this suspension, which was homogenised with orbital shaking at $200 \mathrm{rpm}$ for 120 minutes. The progress of the hydrolysis reaction was monitored by removing a $10 \mu \mathrm{L}$ aliquot from the reaction mixture, diluting with potassium hydroxide solution $(2 \mathrm{~mL}, 0.5 \mathrm{mM}, \mathrm{pH} 10)$, filtering and then recording the UV-Vis spectrum. At each time point, the absorbance at $273 \mathrm{~nm}$ (DMNP reactant) and $400 \mathrm{~nm}$ ( $p$-nitrophenoxide product) were noted. Turn over frequencies were calculated using the half-lives of literature value of GD, and conversion after 15 minutes of DMNP from this work (1.25mg used as $\mathrm{ZrO}_{x}(\mathrm{OH})_{y}$ loading).

\section{Results and discussion}

Synthesis and characterisation of Group IV hydroxylated metal oxides inside carbon nanoreactors

We have developed a two-step synthetic strategy based on the initial encapsulation of the respective metal chlorides in hollow GNF, followed by a hydrolysis step, converting the precursor

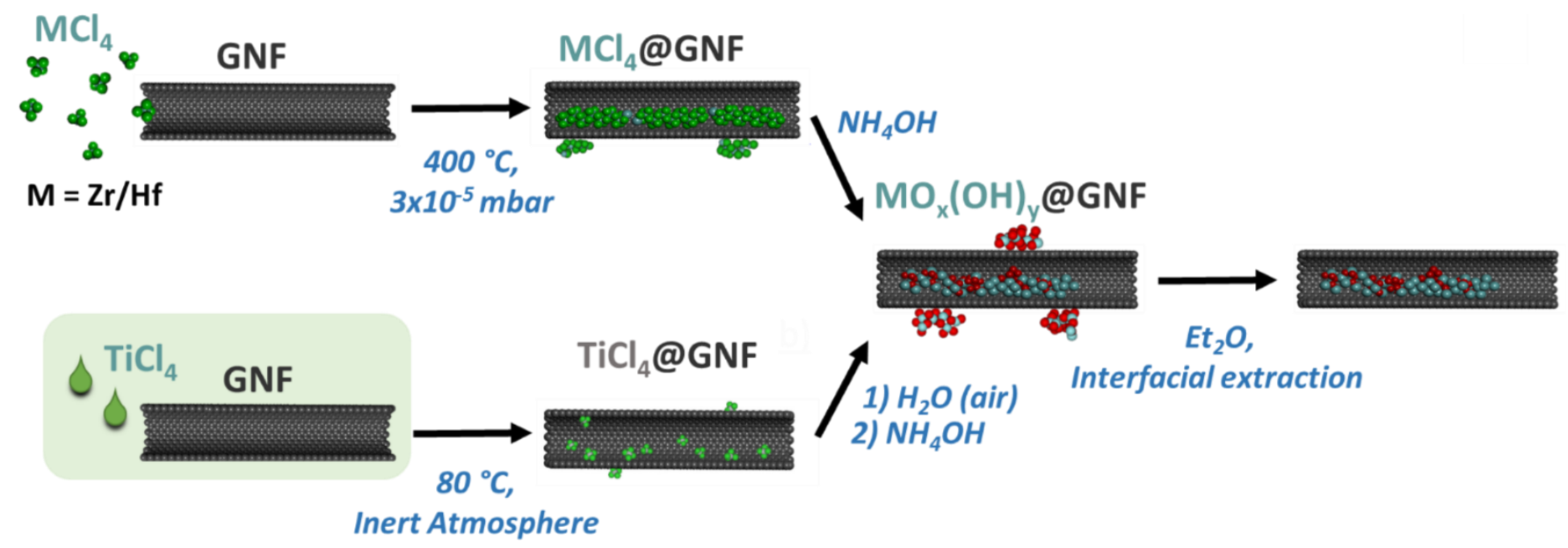

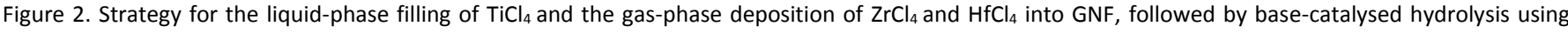
atmospheric moisture and ammonium hydroxide to form the $\mathrm{MO}_{x}(\mathrm{OH})_{y}$ nanomaterial encapsulated by GNF. The interfacial extraction procedure was used to remove externally adsorbed and excess hydrophilic metal oxides within the bulk sample.

nanofibers, GNF $\left(75 \mathrm{mg}\right.$ ) were pre-treated at $500^{\circ} \mathrm{C}$ for $45 \mathrm{~min}$ in air, then immediately loaded into a Pyrex ampoule ( $d=10$ $\mathrm{mm}, \mathrm{L}=12 \mathrm{~cm}$ ) with either $\mathrm{ZrCl}_{4}$ or $\mathrm{HfCl}_{4}(75 \mathrm{mg})$. The solids were then sealed under vacuum $\left(3 \times 10^{-6} \mathrm{mbar}\right)$ and heated at $400{ }^{\circ} \mathrm{C}$ for $48 \mathrm{~h}$. The ampoule was immediately cooled and chloride into a hydroxylated metal oxide (Figure 2). Zirconium and hafnium chlorides $\left(\mathrm{ZrCl}_{4}\right.$ and $\mathrm{HfCl}_{4}$ respectively) were sublimed at $400^{\circ} \mathrm{C}$ into the cavities of open GNF in a vacuum. Upon exposure to water, $\mathrm{ZrCl}_{4}$ and $\mathrm{HfCl}_{4}$ hydrolyse to the corresponding metal oxyhydroxides of the general formula $\mathrm{MO}_{\mathrm{x}}(\mathrm{OH})_{\mathrm{y}}$. The procedure was modified for $\mathrm{TiCl}_{4}$ as, being a 

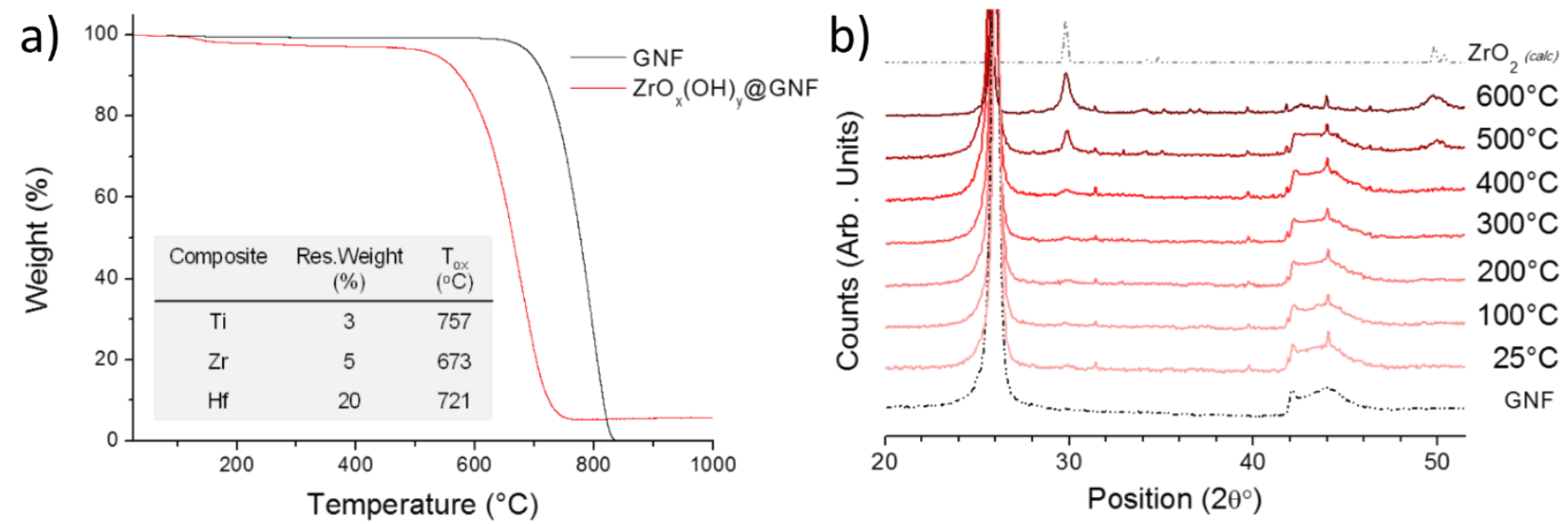

Figure 3. Representative (a) TGA and (b) PXRD analysis of GNF (black) and $\mathrm{ZrO}_{\mathrm{x}}(\mathrm{OH})_{\mathrm{y}} @ \mathrm{GNF}$ (red). TGA allows the metal oxide loading to be calculated based on the residual weight remaining after GNF combustion and provides insight into the interaction of the two components in the composite from the shift in the GNF combustion temperature $\left(T_{o x}\right)$. PXRD initially indicated a lack of crystallinity of the intercalant as only peaks at $2 \theta=26,42$ and $44^{\circ}$ associated with $\mathrm{GNF}$ were significant. Once heated to above $500^{\circ} \mathrm{C}$, a peak at $2 \theta=30^{\circ}$ indicative of $\mathrm{ZrO}_{2}$ appeared, confirming the amorphous nature of the hydroxylated zirconium oxide prior to heating. Persistent peaks at $32^{\circ}$ and $40^{\circ}$ are associated with alumina sample support.

liquid compound with a relatively low surface tension, this precursor compound can be simply filled into GNF by capillary forces, under atmospheric pressure, followed by hydrolysis of $\mathrm{TiCl}_{4} @ \mathrm{GNF}$ into the $\mathrm{TiO}_{\mathrm{x}}(\mathrm{OH})_{\mathrm{y}} @ \mathrm{GNF}$. Due to the reactivity of this precursor, atmospheric moisture is able to hydrolyse this species very quickly. We found the reactions of $\mathrm{MCl}_{4} @ \mathrm{GNF}$ with water to result in incomplete hydrolysis of the metal chloride and partial loss of material from the GNF cavity due to dissolution (Figure S1 - SI). However, the reaction of $\mathrm{MCl}_{4} @ \mathrm{GNF}$ ( $\mathrm{Zr} / \mathrm{Hf})$ with ammonium hydroxide solution, due to the higher $\mathrm{pH}$, resulted in a more complete hydrolysis, thus maximising the yield of surface-hydroxylated metal oxide species inside GNF, as had been shown for related bulk materials previously. ${ }^{36,37}$ For the titanium analogue, the rapid hydrolysis with atmospheric moisture led again to incomplete hydrolysis; however, postwashing with $\mathrm{NH}_{4} \mathrm{OH}$ was used to maximise the surface hydroxyl groups. The abundance of hydroxyl surface groups is important for the catalytic applications of $\mathrm{MO}_{x}(\mathrm{OH})_{y} @ G N F$ in this work and, therefore, $\mathrm{NH}_{4} \mathrm{OH}$ was utilised to aid hydrolysis.

Our strategy offers several benefits compared to other filling methods, such as the direct molten filling of metal oxides, or the encapsulation of pre-formed metal oxide nanoparticles into nanotubes. It requires significantly lower temperatures than molten filling and gives better control over the composition and structure of the final product and is controllable by the conditions (such as $\mathrm{pH}$ ) used in the hydrolysis step. This is not possible in the case of pre-formed nanoparticles, which additionally require a layer of stabilising ligand known to affect their surface chemistry. ${ }^{9,} 38$ Another important benefit of our approach for the synthesis of $\mathrm{MO}_{x}(\mathrm{OH})_{y} @ G N F$ is related to the ability to remove metal oxide that is not encapsulated by GNF. By harnessing the hydrophobic properties of the GNF, once the metal chloride is hydrolysed, an organic solvent can be added to the aqueous suspension, which draws the $\mathrm{MO}_{x}(\mathrm{OH})_{\mathrm{y}} @ \mathrm{GNF}$ composite to the organic-aqueous interface in order to reduce the surface tension and thus leaves any free hydrophilic metal oxide particles remaining in the aqueous phase. This is a new and effective approach allowing purification of the nanoreactors $\mathrm{MO}_{x}(\mathrm{OH})_{y} @ G N F$ from other poorly defined materials that might otherwise be present in the mixture following synthesis, whilst preserving the catalyst inside the GNF. Once isolated, $\mathrm{MO}_{x}(\mathrm{OH})_{y} @ \mathrm{GNF}$ can be utilised for catalysis or processed by a thermal treatment to manipulate their properties for a particular catalytic reaction, opening new avenues for the encapsulation of a wide range of chemically active species into nanoreactors by this method.

Thermogravimetric analysis was employed to confirm the uptake of the precursor molecules by the GNF, with the residual weight after GNF combustion diagnosing the loading (by weight) of the hydroxylated metal oxide in the final composite (Figure 3a). The differences in atomic number, filling method and solubility of the $\mathrm{MO}_{x}(\mathrm{OH})_{y}$ species, critical during the purification step, accounts for the significant changes in loading down the period (Figure S2 - SI). Interestingly, the presence of intercalant in the lumen of the GNF results in a clear decrease in the characteristic GNF oxidation temperature by 40,124 and $76^{\circ} \mathrm{C}$ for $\mathrm{TiO}_{x}(\mathrm{OH})_{y} @ \mathrm{GNF}, \quad \mathrm{ZrO}_{x}(\mathrm{OH})_{y} @ \mathrm{GNF}$ and $\mathrm{HfO}_{x}(\mathrm{OH})_{y} @ G N F$, respectively. This downshift in combustion temperature is associated with electron transfer between the guest-metal species and the host-carbon nanostructure, weakening the carbon-carbon bonding in the graphitic lattice of the GNF sidewalls. The higher Lewis acidic properties of the $\mathrm{Zr}$ composite results in the largest extent of downshifting. Powder $X$-ray diffraction analysis shows no significant crystallinity in any of the prepared $\mathrm{MO}_{x}(\mathrm{OH})_{y} @ \mathrm{GNF}$ samples (Figure 3b), indicating 

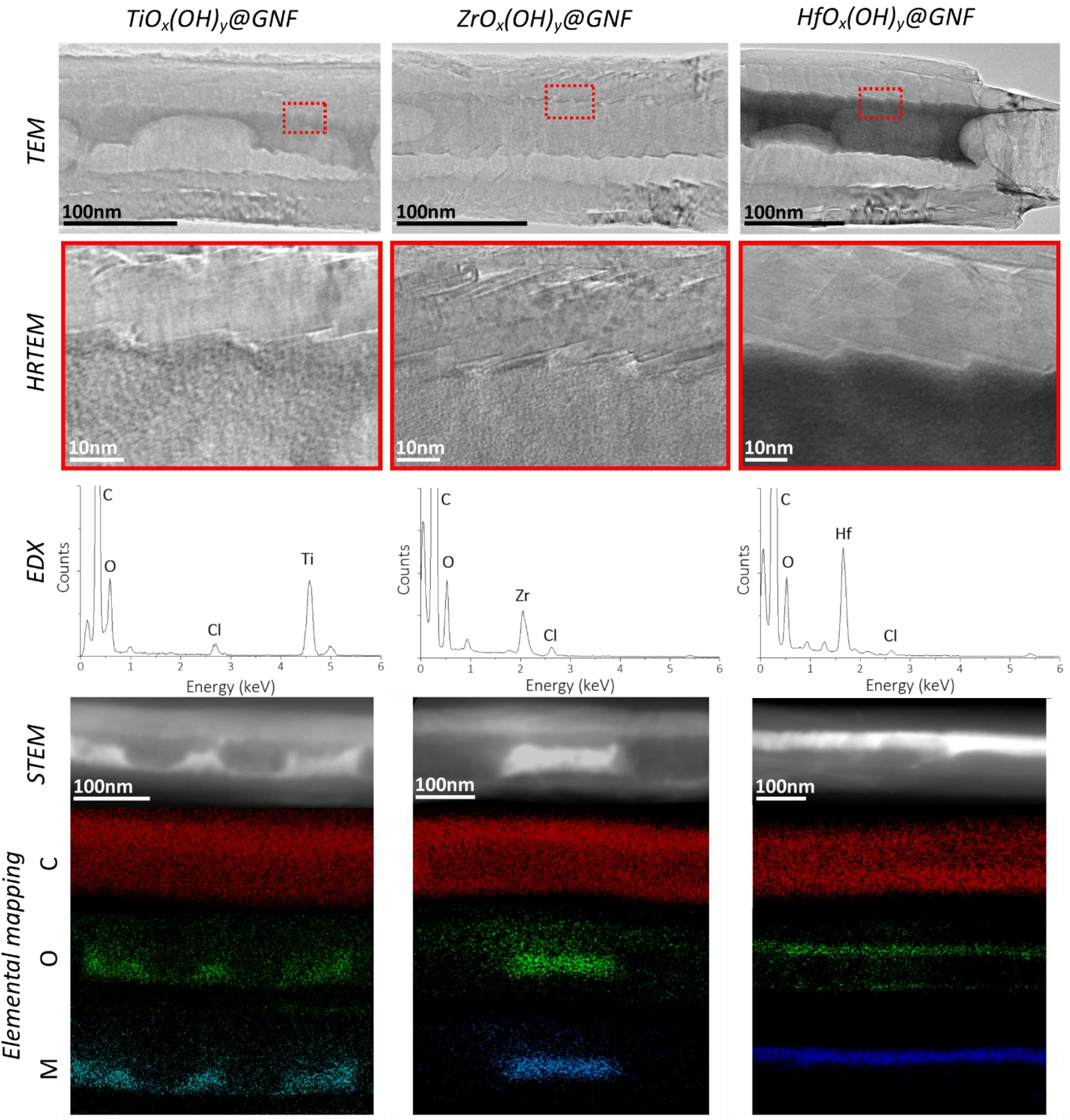

Figure 4. Electron microscopy analysis of $\mathrm{MO}_{x}(\mathrm{OH})_{y} @ G N F$ where $\mathrm{M}=\mathrm{Ti}, \mathrm{Zr}$ and $\mathrm{Hf}$ (left to right). Bright field TEM (where contrast is proportional to $\mathrm{Z}^{0.5}$ ) provides evidence for the position of the hydroxylated metal oxide species within the internal channel of GNF in the composite material. High-resolution TEM micrographs allow for crystalline layers in the GNF to be resolved and highlight the amorphous nature of the encapsulated material templated by the interior step-edges. EDX spectroscopy confirms the presence of the group IV metal within GNF and permits examination of the composition by comparison of the relative atomic percentages. Dark field STEM (where contrast is proportional to $Z^{1.8}$ ) provides clearer contrast between the intercalant and the host, with elemental mapping of the STEM micrographs identifying the respective positions of carbon (red), oxygen (green) and the metal (blue).

either an amorphous phase of hydroxylated metal oxide in GNF or extremely small crystalline domain size. Variable temperature PXRD of the composites indicates crystallisation of the metal oxyhydroxide into a $\mathrm{MO}_{2}$ metal oxide phase at elevated temperature. This can be most clearly observed above $400^{\circ} \mathrm{C}$ for $\mathrm{ZrO}_{x}(\mathrm{OH})_{\mathrm{y}} @ \mathrm{GNF}$, with a tetragonal $\mathrm{ZrO}_{2}$ crystal phase afforded (Figure 3,b), with analogous transitions for the Ti- and $\mathrm{Hf}$-based nanostructures also observed (Figure S3 - SI).

Transmission electron microscopy is the only method that can unambiguously confirm the location, morphology and size of the nanocatalysts within the nanoreactors. ${ }^{39,40}$ Bright field TEM imaging clearly shows the hydroxylated metal oxide encapsulated within the GNF structure, with the image 

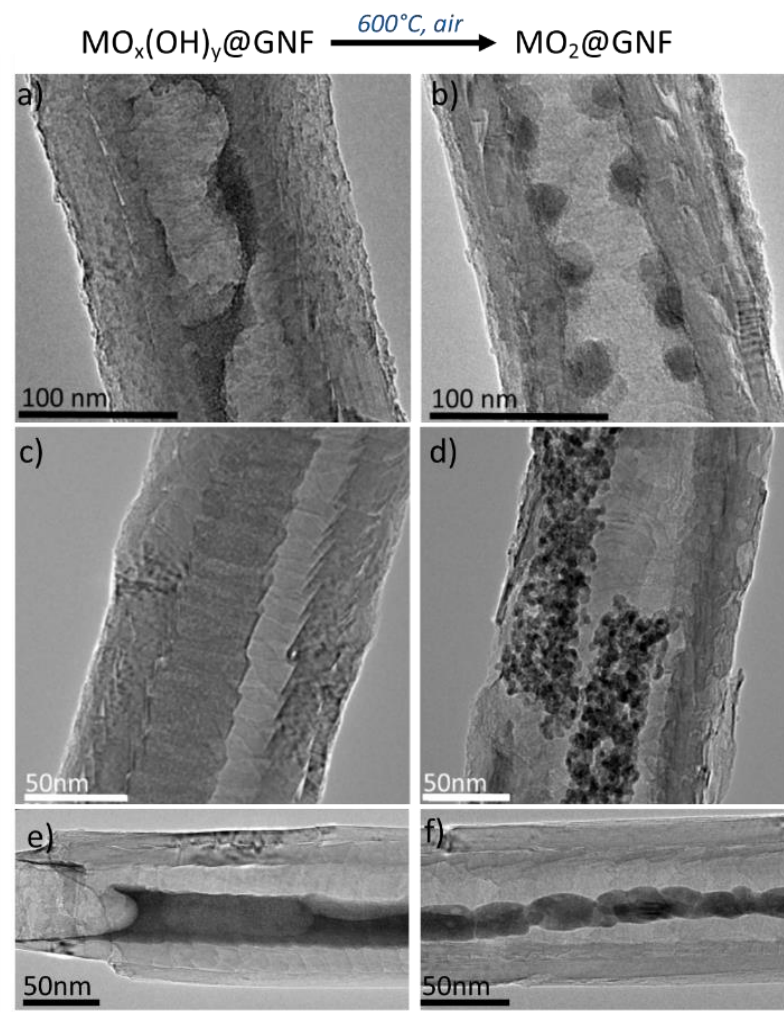

Figure 5. TEM analysis of $\mathrm{MOx}(\mathrm{OH}) \mathrm{y} @ \mathrm{GNF}$ before $(\mathrm{a}, \mathrm{c}, \mathrm{e})$ and after $(\mathrm{b}, \mathrm{d}, \mathrm{f})$ treatment at $600{ }^{\circ} \mathrm{C}$ in air, where $\mathrm{M}=\mathrm{Ti}(\mathrm{a}, \mathrm{b}), \mathrm{Zr}(\mathrm{c}, \mathrm{d})$ and $\mathrm{Hf}(\mathrm{e}, \mathrm{f})$, indicating the formation of discrete metal dioxide nanoparticles after high temperature treatment. This simple process demonstrates that the composition, morphology and size of the encapsulated material can be readily changed and therefore the opportunity to prepare a range of interesting nanocomposite materials.

contrast, though sensitive to several parameters, clearly seen to increase from $\mathrm{Ti}$ to $\mathrm{Zr}$ to $\mathrm{Hf}$ consistent with the increasing atomic numbers of these elements (Figure 4). Surprisingly, instead of forming compact metal oxyhydroxide nanoparticles, $\mathrm{MO}_{\mathrm{x}}(\mathrm{OH})_{\mathrm{y}}$ appear to be coating the interior of the GNF as a thin layer with an average thickness of 6-10 nm, but with substantial variation from one GNF to another. Interestingly, materials treated at $600{ }^{\circ} \mathrm{C}$ in air contained discrete, crystalline $\mathrm{MO}_{2}$ nanoparticles. (Figure 5). High magnification images of the $\mathrm{MO}_{\mathrm{x}} \mathrm{OH}_{\mathrm{y}} @ \mathrm{GNF}$ composites revealed no crystallinity in the metal hydroxides, consistent with the PXRD observations. Lower magnification images indicate no significant variation to the GNF microstructure subsequent to filling with metal oxyhydroxide (Figure $\mathrm{S} 4-\mathrm{SI}$ ).

Energy dispersive $\mathrm{X}$-ray spectroscopy reveals the atomic percentages of the elements present in $\mathrm{MO}_{x}(\mathrm{OH})_{y} @ G N F$. Minimal quantities of chloride detected in the EDX spectra of $\mathrm{MO}_{\mathrm{x}}(\mathrm{OH})_{\mathrm{y}} @ \mathrm{GNF}$ indicate a high conversion of chloride to hydroxide (Figure 4) (Figure S5 and Table S1 - SI). Moreover, the extracted $\mathrm{M}: \mathrm{O}$ ratios are 1:3.5, 1:3.4, and 1:2.5 for $\mathrm{M}=\mathrm{Ti}, \mathrm{Zr}$ and $\mathrm{Hf}$, respectively, corresponding to an approximate formula of $\mathrm{MO}(\mathrm{OH})_{2}$ for $\mathrm{Ti}$ and $\mathrm{Zr}$. This indicates that these metals are in their expected +4 oxidation state, as confirmed by electron energy loss spectroscopy (Figure S6 - SI), and that hydroxyl groups are produced during the hydrolysis of metal chlorides inside the GNF (Table S1 - SI). ${ }^{41}$ Due to the preferred form of hafnium oxide, an approximate formula of $\mathrm{HfO}_{2}$ is proposed. The non-stoichiometric composition of the hydrolysis products is likely to be responsible for the lack of crystalline order, which allows the metal hydroxides to spread over the inner surface of GNF (rather than to form compact crystalline particles), following the contours of the step-edges, thus maximising the surface area available for catalysis.

To confirm the increased surface area for $\mathrm{ZrO}(\mathrm{OH})_{2} @ \mathrm{GNF}$, Brunauer-Emmett-Teller analysis showed a $13 \mathrm{~m}^{2} / \mathrm{g}$ increase in the surface area in comparison to empty GNFs. Considering that the hydroxylated metal oxide comprises only $5 \mathrm{wt} \%$ of $\mathrm{ZrO}_{\mathrm{x}}(\mathrm{OH})_{\mathrm{y}} @ \mathrm{GNF}$, this indicates that the accessible surface area of encapsulated $\mathrm{ZrO}_{x}(\mathrm{OH})_{y}$ is $141 \mathrm{~m}^{2} / \mathrm{g}$, which is four times higher than that of the metal hydroxide synthesised in the absence of GNF (Table S2 - SI). In addition, BET measurements provide information about the pore volume in $\mathrm{ZrO}_{x}(\mathrm{OH})_{y} @ G N F$, estimating the accessible volume to be $\sim 0.08 \mathrm{~cm}^{3} / \mathrm{g}$ in the nanoreactors, indicating that no blockages exist and thus GNF possess an effective space for confined reactions.
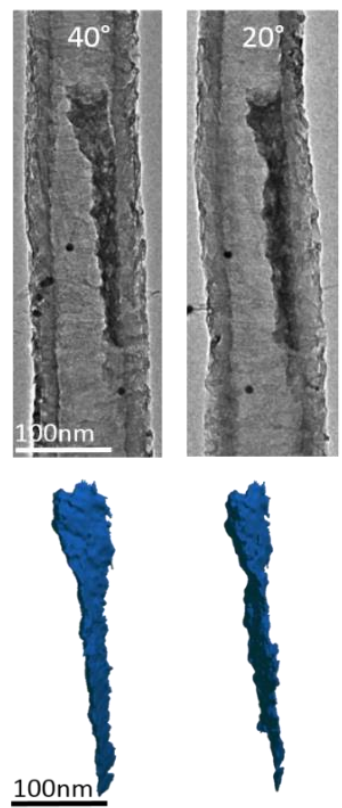
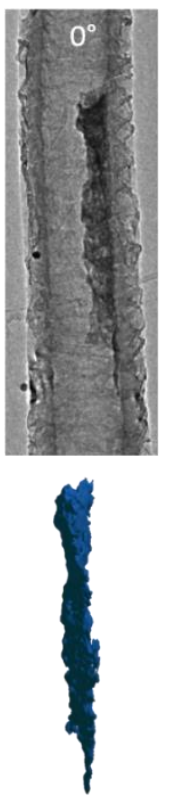
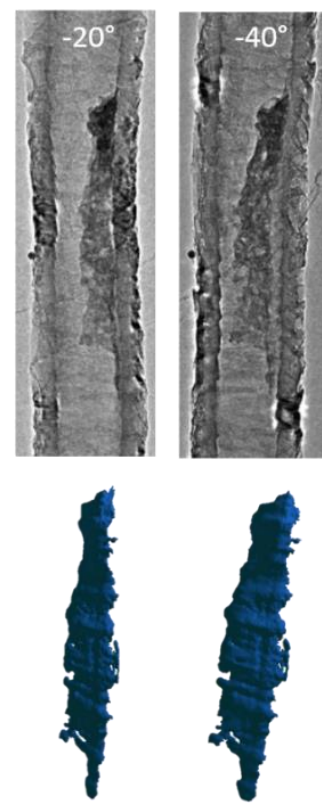

Figure 6. Bright field TEM tilt series (top) of $\mathrm{ZrO}(\mathrm{OH})_{2}$. The 3D model (bottom) of this species was reconstructed from the bright field and dark field STEM images (Figure S7 - SI), which accentuate the contrast of the intercalant and highlight the thin layer coating on the interior surface, which has many topological defects and edges. Au fiducial markers were used to assist tomography and reconstruction. 
a)

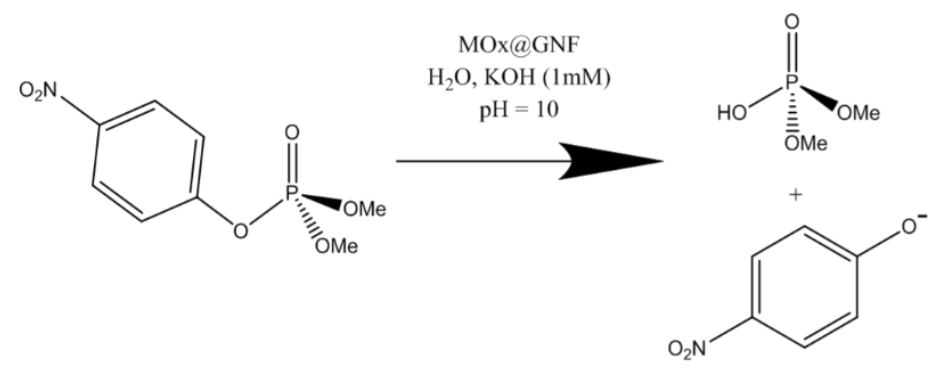

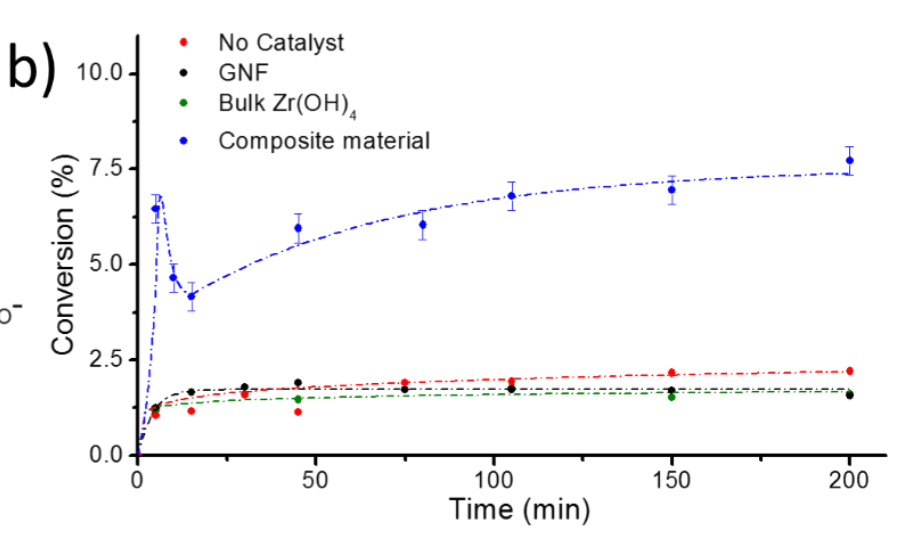

Figure 7. (a) Scheme for the hydrolysis of the simulant DMNP with the composite material, which can be monitored by UV-vis spectroscopy using the diagnostic peaks at 400 and $273 \mathrm{~nm}$ for the starting material and product, respectively. (b) The time-dependence of hydrolysis of DMNP highlights the enhanced hydrolysis attained using the $\mathrm{ZrO}(\mathrm{OH})_{2} @ G N F$ composite nanoscale material relative to control reactions using GNF, bulk hydroxylated zirconia and in the absence of a catalyst.

Dark field scanning transmission electron microscopy allows better differentiation of image contrast between carbon and the heavier elements as the contrast intensity is proportional to the $Z^{1.8}$ in dark field STEM as compared to $Z^{0.5}$ for bright field TEM (Figure 4). EDX spectroscopic mapping in STEM mode further supports the location of metal hydroxides inside GNF and also links the high contrast in TEM images with the presence of $\mathrm{Ti}, \mathrm{Zr}$ and $\mathrm{Hf}$ within $\mathrm{MO}_{\mathrm{x}}(\mathrm{OH})_{\mathrm{y}} @ \mathrm{GNF}$. Furthermore, the distribution of oxygen correlates well with the distribution of metal atoms in GNF, indicating that the oxygen signals in the EDX spectra are predominantly associated with $\mathrm{O}$ atoms of metal hydroxides/oxides, rather than oxygen-containing surface groups of the GNF for example, validating the quantitative EDX spectroscopy analysis described (Figure 4).

3D analysis of $\mathrm{ZrO}(\mathrm{OH})_{2} @ G N F$ was achieved using TEM tomography (Figure 6). Both bright field TEM and dark field STEM tomography series were recorded by tilting the TEM grids around the GNF growth axis and revealed that the hydroxylated metal oxide layers did not cover the entire interior surface, but just under half of the interior side wall (Figure 6) (Figure S7 - SI). TEM imaging and tomography of other group IV metals also reveal the structures of the $\mathrm{MO}_{x}(\mathrm{OH})_{y}$ layers to be uneven, exhibiting significant topological defects, which have potential as reactive sites for decontamination reactions (Figure S8 - SI).

Hydrolysis of dimethyl nitrophenyl phosphate using encapsulated hydroxylated zirconium in carbon nanoreactors To explore the properties of the catalytic nanoreactors in the hydrolysis of organophosphates, dimethyl nitrophenyl phosphate (DMNP) was utilised. DMNP is often used to study organophosphate decontamination and has the added advantage of forming $p$-nitrophenoxide during hydrolysis, which allows the kinetics of this reaction to be readily monitored using UV-Vis spectroscopy (Figure 7). $\mathrm{ZrO}(\mathrm{OH})_{2} @ \mathrm{GNF}$ was tested as a catalyst for DMNP hydrolysis and found to be highly effective, consistent with previous reports for related zirconium-based hydroxides, outperforming the other group IV materials prepared in our study, owing to the maximised Lewis acidic properties and increased surface hydroxyl groups. ${ }^{24,28,42}$ Moreover, the rate of hydrolysis promoted by $\mathrm{ZrO}(\mathrm{OH})_{2} @ G N F$ when compared with separate hydroxylated zirconia and empty GNF revealed that $\mathrm{ZrO}(\mathrm{OH})_{2} @ \mathrm{GNF}$ is significantly more active (Figure 7).

Considering the small percentage of the catalytically active material in $\mathrm{ZrO}(\mathrm{OH})_{2} @ G N F$ to the high quantity of simulant present in solution (3.6 mol\%) the catalytic nanoreactors have shown impressive initial decontamination abilities towards the organophosphate. A turn over frequency (TOF) for DMNP hydrolysis of $1.3 \times 10^{-2} \mathrm{~min}^{-1}$ was calculated, which exceeds the TOF for Sarin obtained using bulk zirconium hydroxide by a factor of $12 . .^{28}$ In addition, it is important to emphasise that individually GNF and bulk hydroxylated zirconia (Figure S9 - SI) have little impact on the hydrolysis rate of DMNP (relative to control reactions); however, when combined in $\mathrm{ZrO}(\mathrm{OH})_{2} @ \mathrm{GNF}$, the synergistic effectiveness of the components is enhanced 4-fold. The origin of these synergistic effects relate to the unusual topography and composition of the confined nanocatalyst and the enhanced host-guest interactions between reactant molecules and the GNF interior surfaces. In particular, the increased surface area of the metal species coating smeared over the GNF internal surface and the presence of structural defects in the non-stoichiometric hydroxide can lead to increased Lewis acidity and accessibility of the active sites in $\mathrm{ZrO}(\mathrm{OH})_{2} @ G N F$ which are important for the hydrolysis of OP agents (Figure 8).43 Interestingly, after the calcination of $\mathrm{ZrO}(\mathrm{OH})_{2} @ \mathrm{GNF}$ at $600{ }^{\circ} \mathrm{C}$, which leads to more structured and crystalline $\mathrm{ZrO}_{2} @ G N F$ (Figure $5 \mathrm{~d}$ ), the catalytic effects on the hydrolysis pathway are lost (Figure S10 - SI), thus confirming the importance of a non-crystalline structured catalyst and the presence of the surface $\mathrm{OH}$-groups. Furthermore, confinement of a catalyst in a nanoreactor is 
a)

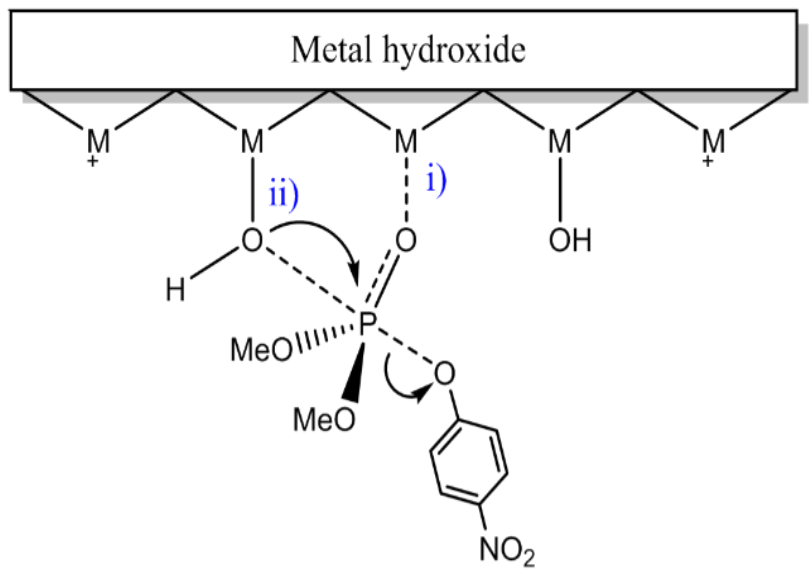

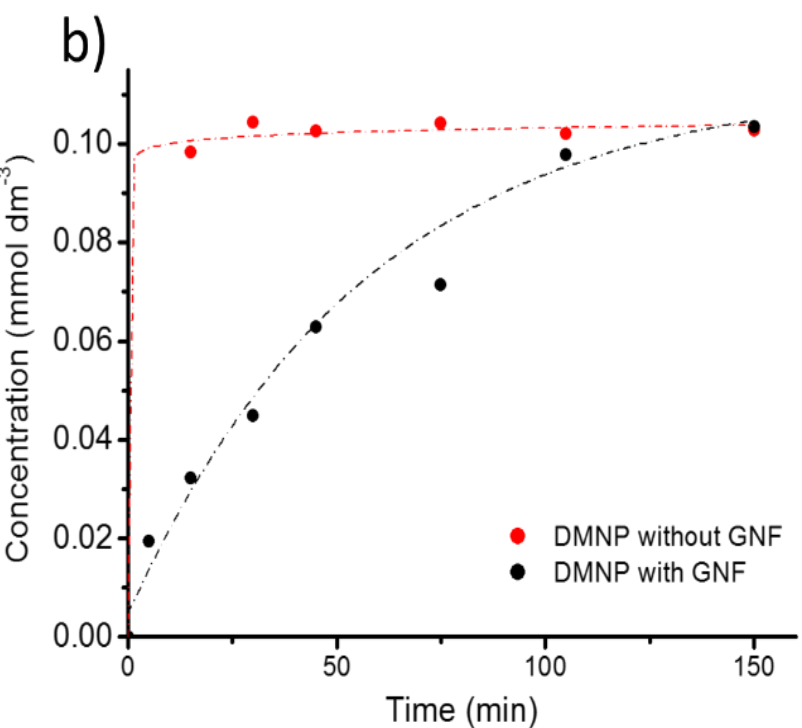

Figure 8. (a) The recognised mechanism for the hydrolysis of phosphate esters using group IV metal oxides, involving (i) the coordination of the phosphate group with metal cations and (ii) nucleophilic attack of surface hydroxyl groups. ${ }^{36}$ (b) The change in DMNP concentration profile in the presence of GNF highlights the ability of GNF to act as 'nano-sponges', rapidly taking up, but slowly releasing DMNP over time. This is particularly striking as GNF exhibit no change in volume and possess minimal flexibility - features typically associated with sponge-like adsorbents - and represents a key feature for efficient DMNP hydrolysis inside GNF.

known to enhance local concentration of reactants around catalytic centres, and may also contribute to the increased rate of hydrolysis observed for $\mathrm{ZrO}_{\mathrm{x}}(\mathrm{OH})_{\mathrm{y}} @ \mathrm{GNF} .{ }^{44}$

The observed kinetics of DMNP hydrolysis in $\mathrm{ZrO}(\mathrm{OH})_{2} @ \mathrm{GNF}$ nanoreactors indicates an unusual feature within the first 20 minutes of the reaction: a rapid initial increase of the conversion rate, followed by a drop after 5 minutes and subsequent gradual rate increase after 20 minutes. This may indicate that in the first few minutes the reactant concentration changes not only due to hydrolysis but also as a consequence of some additional faster processes, such as the physical absorption of DMNP into the GNF cavity. To investigate this phenomenon, a control experiment monitoring the concentration of DMNP was performed with and without the GNF (Figure 8). These measurements show that GNF are able to physically and reversibly remove DMNP from aqueous solution without hydrolysis, which is subsequently released back into the solution from GNF over time. The same effect is responsible for the increased concentration of reactants in nanotubes, as discussed. Previous work utilising carbon nanoreactors for catalysis has identified local concentrations as key for confined reactions. ${ }^{39}$ In the context of the DMNP hydrolysis with $\mathrm{ZrO}(\mathrm{OH})_{2} @ \mathrm{GNF}$, the observed 'nano-sponge' effect is likely to be responsible for the spike in the conversion curves based on the rapid absorption and release of the starting material compared to the phenolate production.

The initially rapid hydrolysis observed using $\mathrm{ZrO}(\mathrm{OH})_{2} @ G N F$ does subside over time, presumably due to catalyst poisoning and loss of the hydroxyl surface groups. Having the phosphate irreversibly binding to the surface reduces the number of Lewis acidic sites available for hydrolysis. TGA indicates minimal catalyst loss after hydrolysis, providing promising results regarding the stability of these materials within the nanoreactors, but also shows a reduction in the Lewis acidic properties of the catalysts, due to either binding of the OP species or a decrease in the accessible hydroxyl surface groups (Figure S11 - SI). TEM and EDX spectroscopy after the decontamination reaction suggests that the catalyst remains within the carbon nanostructure throughout the course of the reaction, but also shows the presence of phosphorus in this structure (Figure S12 - SI). Therefore, removing the surfaceadsorbed organophosphates from the catalyst is an important challenge, a feat which has been previously achieved by applying a large excess of metal hydroxide. ${ }^{28}$ In our study, spatial confinement was employed to optimise and retain the catalytic performance of the metal hydroxides. Regeneration of catalytic activity in nanoreactors is the next challenge to address.

\section{Conclusions}

A general strategy has been established for the encapsulation of group IV metal chlorides inside graphitised nanofibers which can then be easily manipulated to change the composition or morphology to produce interesting and unique confined nanostructures. The effective filling of hydroxylated group IV metal oxides into GNF has been demonstrated using gas phase deposition or liquid filling of the metal chloride precursor followed by hydrolysis with ammonium hydroxide or atmospheric moisture. The excess metal oxide can be effectively removed by exploiting the hydrophobic nature of the GNF producing a composite material with significant synergistic electronic interactions between the carbon nanostructure and its intercalant. The GNF are able to template a unique thin layer 
amorphous coating which covers large portions of the nanofiber interior channel due to preferential interaction with the GNF step-edges. This encapsulated nanomaterial has both a high surface area and a large number of reactive topological edges which proves crucial for decontamination catalysis. The hydroxylated zirconia nanoreactor has therefore shown rapid initial decontamination abilities when applied to chemical warfare agent simulant hydrolysis, with a 4-fold enhancement relative to its constituent components observed. It has also been clearly demonstrated that GNF act not only as a nanocontainer but are also instrumental in effective sequestration of the DMNP simulant from aqueous solutions and have been identified as promising candidates for toxic OP 'nano-sponges.' This effective absorption in the presence of the nanoscale hydroxylated zirconia is thought to be one of the key driving forces behind the increased activity of the catalyst and, although surface deactivation and poisoning remain a challenge, the ability of the catalysts to remain within the nanoreactor after decontamination is advantageous. This work highlights the benefits of continuing catalysts within carbon nanostructures to form nanoreactors, utilising their unique hydrophobic 'nano-sponge' properties to absorb reagents of interest, leading to enhanced reaction kinetics.

\section{Conflicts of interest}

There are no conflicts to declare.

\section{Acknowledgements}

The authors would like to thank the University of Nottingham and the Engineering and Physical Science Research Council (EPSRC) for the funding which supported this project (EP/L022494/1). Thanks are also given to the Nanoscale and Microscale Research Centre for access to the microscopy facilities, Julie Watts for the SEM imaging and Alison Taylor for assistance with the BET measurements.

\section{Notes and references}

1. J. J. Shi, Y. Y. Wang, W. C. Du and Z. Y. Hou, Carbon, 2016, 99, 330-337.

2. H. B. Zhang, X. L. Pan and X. H. Bao, J. Energy Chem., 2013, 22, 251-256.

3. P. Serp and E. Castillejos, ChemCatChem, 2010, 2, 41-47.

4. A. N. Khlobystov, D. A. Britz and G. A. D. Briggs, Acc. Chem. Res., 2005, 38, 901-909.

5. R. S. Ruoff and D. C. Lorents, Carbon, 1995, 33, 925-930.

6. K. Balasubramanian and M. Burghard, Small, 2005, 1, 180192.

7. X. L. Pan and X. H. Bao, Acc. Chem. Res., 2011, 44, 553-562.

8. O. N. Metelkina, R. W. Lodge, P. G. Rudakovskaya, V. M. Gerasimov, C. H. Lucas, I. S. Grebennikov, I. V. Shchetinin, A. G. Savchenko, G. E. Pavlovskaya, G. A. Rance, M. D. Gimenez-Lopez, A. N. Khlobystov and A. G. Majouga, Journal of Materials Chemistry C, 2017, 5, 2167-2174.

9. M. D. Gimenez-Lopez, A. La Torre, M. W. Fay, P. D. Brown and A. N. Khlobystov, Angewandte Chemie-International Edition, 2013, 52, 2051-2054.
10. M. A. Lebedeva, T. W. Chamberlain, M. Schroder and A. N. Khlobystov, Chem. Mater., 2014, 26, 6461-6466.

11. B. Cornelio, A. R. Saunders, W. A. Solomonsz, M. LaronzeCochard, A. Fontana, J. Sapi, A. N. Khlobystov and G. A. Rance, J. Mater. Chem., 2015, 3, 3918-3927.

12. E. Castillejos, P. J. Debouttiere, L. Roiban, A. Solhy, V. Martinez, Y. Kihn, O. Ersen, K. Philippot, B. Chaudret and P. Serp, Angew. Chem.-Int. Edit., 2009, 48, 2529-2533.

13. A. Botos, J. Biskupek, T. W. Chamberlain, G. A. Rance, C. T. Stoppiello, J. Sloan, Z. Liu, K. Suenaga, U. Kaiser and A. N. Khlobystov, J. Am. Chem. Soc., 2016, 138, 8175-8183.

14. T. W. Chamberlain, A. M. Popov, A. A. Knizhnik, G. E. Samoilov and A. N. Khlobystov, ACS nano, 2010, 4, 52035210.

15. C. T. Stoppiello, J. Biskupek, Z. Y. Li, G. A. Rance, A. Botos, R. M. Fogarty, R. A. Bourne, J. Yuan, K. R. J. Lovelock, P. Thompson, M. W. Fay, U. Kaiser, T. W. Chamberlain and A. N. Khlobystov, Nanoscale, 2017, 9, 14385-14394.

16. A. La Torre, M. D. Gimenez-Lopez, M. W. Fay, G. A. Rance, W. A. Solomonsz, T. W. Chamberlain, P. D. Brown and A. N. Khlobystov, ACS nano, 2012, 6, 2000-2007.

17. M. V. Kharlamova, Appl. Phys. A-Mater. Sci. Process., 2016, $122,8$.

18. Y. Bai, Y. B. Dou, L. H. Xie, W. Rutledge, J. R. Li and H. C. Zhou, Chem. Soc. Rev., 2016, 45, 2327-2367.

19. Y. M. Dai, L. L. Niu, J. Q. Zou, T. X. Chen, H. Liu and Y. Zhou, Chin. Chem. Lett., 2018, 29, 887-891.

20. X. Y. Xiao, L. L. Liu, J. H. Ma, Y. Ren, X. W. Cheng, Y. H. Zhu, D. Y. Zhao, A. A. Elzatahry, A. Alghamdi and Y. H. Deng, ACS Appl. Mater. Interfaces, 2018, 10, 1871-1880.

21. M. N. Zhang, J. Jia, K. Huang, X. D. Hou and C. B. Zheng, Chin. Chem. Lett., 2018, 29, 456-460.

22. M. J. Katz, J. E. Mondloch, R. K. Totten, J. K. Park, S. T. Nguyen, O. K. Farha and J. T. Hupp, Angew. Chem. Int. Ed. Engl., 2014, 53, 497-501.

23. N. E. Thornburg, A. B. Thompson and J. M. Notestein, Acs Catalysis, 2015, 5, 5077-5088.

24. J. E. Mondloch, M. J. Katz, W. C. Isley, 3rd, P. Ghosh, P. Liao, W. Bury, G. W. Wagner, M. G. Hall, J. B. DeCoste, G. W. Peterson, R. Q. Snurr, C. J. Cramer, J. T. Hupp and O. K. Farha, Nat. Mater., 2015, 14, 512-516.

25. N. Sharma and R. Kakkar, Adv. Mat. Lett, 2013, 4, 508-521.

26. J. H. Choi, Y. Mao and J. P. Chang, Mater. Sci. Eng. R-Rep., 2011, 72, 97-136.

27. I. Salem, Catal. Rev.-Sci. Eng., 2003, 45, 205-296.

28. T. J. Bandosz, M. Laskoski, J. Mahle, G. Mogilevsky, G. W. Peterson, J. A. Rossin and G. W. Wagner, J Phys Chem C, 2012, 116, 11606-11614.

29. V. V. Singh, A. Martin, K. Kaufmann, S. D. S. de Oliveira and J. Wang, Chem. Mater., 2015, 27, 8162-8169.

30. T. Islamoglu, A. Atilgan, S. Y. Moon, G. W. Peterson, J. B. DeCoste, M. Hall, J. T. Hupp and O. K. Farha, Chem. Mater., 2017, 29, 2672-2675.

31. P. W. Elsinghorst, F. Worek and M. Koller, Toxicol. Lett., 2015, 233, 207-213.

32. Q. F. Yang, J. Wang, W. T. Zhang, F. B. Liu, X. Y. Yue, Y. N. Liu, M. Yang, Z. H. Li and J. L. Wang, Chem. Eng. J., 2017, 313, 19-26.

33. F. M. Raushel, Nature, 2011, 469, 310-311.

34. G. M. Kazankov, V. S. Sergeeva, E. N. Efremenko, L. Alexandrova, S. D. Varfolomeev and A. D. Ryabov, Angew. Chem.-Int. Edit., 2000, 39, 3117-3119.

35. D. N. Mastronarde, J. Struct. Biol., 1997, 120, 343-352. 
36. D. A. Giannakoudakis, J. K. Mitchell and T. J. Bandosz, J. Mater. Chem. A, 2016, 4, 1008-1019.

37. G. K. Chuah, S. Jaenicke, S. A. Cheong and K. S. Chan, Appl. Catal. A-Gen., 1996, 145, 267-284.

38. Y. K. Chen, A. Chu, J. Cook, M. L. H. Green, P. J. F. Harris, R. Heesom, M. Humphries, J. Sloan, S. C. Tsang and J. F. C. Turner, J. Mater. Chem., 1997, 7, 545-549.

39. W. A. Solomonsz, G. A. Rance, B. J. Harris and A. N. Khlobystov, Nanoscale, 2013, 5, 12200-12205.

40. S. A. Miners, G. A. Rance and A. N. Khlobystov, Chem. Soc. Rev., 2016, 45, 4727-4746.

41. C. Ahn and O. Krivanek, EELS atlas, Gatan, 1983.

42. I. V. Schweigert and D. Gunlycke, J. Phys. Chem. A, 2017, 121, 7690-7696.

43. Y. J. Jang, K. Kim, O. G. Tsay, D. A. Atwood and D. G. Churchill, Chem. Rev., 2015, 115, PR1-76.

44. A. N. Khlobystov, ACS nano, 2011, 5, 9306-9312. 